\title{
Non-random distribution of methyl-CpG sites and non-CpG methylation in the human rDNA promoter identified by next generation bisulfite sequencing
} Running title: DNA methylation patterns of the human rDNA promoter

\begin{abstract}
Maciej Pietrzak $^{1 \#}$, Grzegorz A. Rempala ${ }^{3}$, Peter T. Nelson ${ }^{4}$, Michal Hetman $^{1,2, \text { II }}$
${ }^{1}$ Kentucky Spinal Cord Injury Research Center and the Departments of Neurological Surgery and ${ }^{2}$ Pharmacology\&Toxicology, University of Louisville, Louisville, Kentucky 40292, ${ }^{3}$ Division of Biostatistics and Mathematical Biosciences Institute, The Ohio State University, Columbus, Ohio, 43210, ${ }^{4}$ Sanders-Brown Center on Aging, University of Kentucky, Lexington, Kentucky 40536
\end{abstract}

\# Present address: Division of Biostatistics, College of Public Health and Center for Pharmacogenomics, College of Medicine, The Ohio State University, Columbus, Ohio, 43210

II To whom correspondence should be addressed: Dr. Michal Hetman, KY Spinal Cord Injury Research Center, University of Louisville, 511 S. Floyd St., MDR616, Louisville, KY 40292. Tel.: 502-852-3619; Fax: 502-852-5148; e-mail: michal.hetman@louisville.edu 


\begin{abstract}
A next generation bisulfite sequencing (NGBS) was used to study rDNA promoter methylation in human brain using postmortem samples of the parietal cortex. Qualitative analysis of patterns of $\mathrm{CpG}$ methylation was performed at the individual rDNA unit level. $\mathrm{CpG}$ sitespecific differences in methylation frequency were observed with the core promoter harboring three out of four most methylated CpGs. Moreover, there was an overall trend towards comethylation for all possible pairs of $26 \mathrm{CpG}$ sites. The hypermethylated $\mathrm{CpGs}$ from the core promoter were also most likely to be co-methylated. Finally, although rare, non-CpG $(\mathrm{CpH})$ methylation was detected at several sites with one of them confirmed using the PspGI-qPCR assay. Similar trends were observed in samples from control individuals as well as patients suffering of Alzheimer's disease (AD), mild cognitive impairment (MCI) or ataxia telangiectasia (AT). Taken together, while some methyl-CpG sites including those in the core promoter may have relatively greater inhibitory effect on rRNA transcription, co-methylation at multiple sites may be required for full and/or long lasting silencing of human rDNA.
\end{abstract}

Keywords: neurodegeneration, rDNA, nucleolus, DNA methylation, brain, aging 


\section{Introduction}

Dynamic methylation of $26 \mathrm{CpG}$ sites in the promoters of the repeated human nucleolar rRNA genes (ribosomal DNA, rDNA) alters the pool of transcriptionally active units (McStay \& Grummt 2008, Brown \& Szyf 2007). Transcriptionally active rDNA contains hypomethylated CpGs throughout the promoter (Brown \& Szyf 2007). Conversely, the nucleolar repressive complex (NoRC) increases $\mathrm{CpG}$ methylation as well as repressive histone modifications inducing formation of silent heterochromatic rDNA and subsequently reducing ribosomal biogenesis (Guetg et al. 2010).

While transient reduction of ribosomal biogenesis may be beneficial by improving cell survival under stress, its prolonged disruption may negatively affect translation-intensive processes such as proliferative cell growth or neuronal morphogenesis (Murayama et al. 2008, Freed et al. 2010, Slomnicki et al. 2016). Although rate of ribosomal biogenesis declines as neurons become terminally post-mitotic, they keep expanding their ribosome content (Slomnicki et al. 2016). As a result, at least during the maturation period, perturbed ribosomal biogenesis affects neuronal ribosome numbers, translation and dendritic tree development (Slomnicki et al. 2016).

Nucleolar dysfunction has been reported in several neurodegenerative diseases including Alzheimer's disease (AD), Parkinson's disease and Huntington's disease (Hetman \& Pietrzak 2012). For example, in the cerebral cortex, ribosomal deficits were found in AD as well as mild cognitive impairment (MCI) which often represents early stage AD (Ding et al. 2005, Ding et al. 2006). Such deficits were accompanied by increased methyl-CpG (mCpG) content in the rDNA promoter (Pietrzak et al. 2011). Therefore, epigenetic silencing of rDNA may contribute to AD- 
associated ribosomal deficiency, and, in consequence, synapse loss and dementia, or, be part of the secondary brain tissue response to neurodegenerative stress.

AD-associated changes of rDNA methylation were studied using bisulfite sequencing (Pietrzak et al. 2011). While this method provides data on DNA methylation at a single CpG level, it is limited by a relatively small number of reads that can be obtained in a timely manner (typically 10-30/sample). Such small-scale sampling is of particular concerns when studying methylation of multicopy genes with multiple methylation sites. Indeed, limited sampling is perhaps the primary reason why complex patterns of human rDNA methylation have never been a subject of detailed analysis. To bypass such a limitation, we have developed a next generation bisulfite sequencing (NGBS)-based approach to analyze methylation of the human rDNA promoter. Using this method we analyzed patterns of DNA methylation at the individual rDNA unit level using post-mortem brain samples from patients with MCI, AD, AT and age-matched controls.

\section{Materials and Methods}

2.1 Subjects and sample preparation. The AD-, MCI- and their age-matched control samples were from donors who were participants of the IRB-approved University of Kentucky AD Center cohort and were followed for at least 2 years before death (Nelson et al. 2007). Tissue samples of parietal cortex (Brodmann Areas 39 and 40) were flash-frozen in liquid nitrogen ( 5 or less hours after death) and stored at $-80^{\circ} \mathrm{C}$. Unfixed, freshly frozen brain tissue samples of donors with AT and their age-matched controls were obtained from the NICHD Brain and Tissue Bank for Developmental Disorders (University of Maryland). None of the control donors for the AT study was diagnosed with a chronic neurological disease. Summary of donor 
data is listed in Table 1.

2.2 Bisulfite mapping of rDNA methylation. A bisulfite conversion of genomic DNA was performed as described previously using EZ DNA Methylation-Direct Kit (Zymo Research), (Pietrzak et al. 2011). The rDNA promoter fragment containing 26 potential mCpGs was PCR amplified. As $\mathrm{CpG}$ methylation estimation may be biased by methyl-sensitive PCR priming (Fuso et al. 2015), we used primers that localized in the regions lacking CpG sites. The primes contained unique short tags (Multiplex Identifiers or MIDs) that were specific for each subject. To ensure accuracy of tag sequencing, we used a set of 10-base Extended Multiplex Identifiers optimized for multiplexing in 454 Sequencing system (Roche 454 Sequencing Technical Bulletin No. 005-2009, primer information details presented in Table S1).

2.3 Amplicon sequencing and analysis. PCR products from 46 samples were pooled in equimolar amounts and sequenced using 454 GS Junior System (Roche, Indianapolis, IN) at Cofactor Genomic, St Louis, MO. The emulsion PCR and subsequent sequencing reaction were performed exactly as described in the GS Junior emPCR Method Manual (Roche, Mannheim, Germany). Read quality assessment was performed using FastQC version 0.10.0 (Andrews, 2010, available online at: http://www.bioinformatics.babraham.ac.uk/projects/fastqc). Qualityfiltered sequencing reads were decoded and reads containing full-length rDNA promoter sequence were binned based on unique tags for each subject using a custom built script. The rDNA promoter methylation was analyzed with BIQ AnalyzerHT (http://biq-analyzerht.bioinf.mpi-inf.mpg.de) (Lutsik et al. 2011). Binary representation of $\mathrm{mC}$ in sequencing reads (1-methylation, 0-no methylation at a given site) was exported from BIQ AnalyzerHT for further analysis as described below.

2.4 Analysis of putative non- CpG methylation sites. To detect non-canonical methylation 
sites in human brain rDNA gene promoter, total pools of reads from analyzed groups were screened for unconverted $\mathrm{C}$ at $\mathrm{CpH}$ sites with BIQ AnalyzerHT. For each site percentage of nonconversion was calculated. To filter the results against possible conversion errors, only those sites with non-conversion frequencies that were higher than the average frequency of $\mathrm{CpH}$ nonconversion across the entire region were considered. In order to be identified as candidate target of $\mathrm{CpH}$ methylation, a $\mathrm{CpH}$ sites had to reach the above average non-conversion frequency in at least four of five rDNA pools that were investigated.

\subsection{Methyl-sensitive restriction enzyme-qPCR analysis of methylation. Methylation-}

sensitive restriction analysis was performed as described previously (Pietrzak et al. 2011). After HpaII digestion of genomic DNA the rDNA promoter fragment containing a potential mCpG in a HpaII recognition site (positions -11 to -8 from the transcription start site, primer details are in Table S1) was amplified by qPCR; an HpaII-uncleavable fragment of rDNA was used as a normalizer. The qPCR data were analyzed using the standard curve method. To confirm methylation of a putative $\mathrm{CpH}$ site at position -94, $200 \mathrm{ng}$ of DNA were treated with PspGI (New England Biolabs, Ipswitch, MA) for six hours at $75^{\circ} \mathrm{C}$ as this $\mathrm{CpH}$ is a part of a recognition site for PspGI (positions -95 to -91). A fragment containing this site was then amplified by qPCR using flanking primers. For a negative control, PspGI-treated genomic DNA template was cut with MspI (New England Biolabs, Ipswitch, MA) for six hours at $37^{\circ} \mathrm{C}$. As several MspI sites were present in the analyzed amplicon, MspI treatment was expected to prevent amplification. Non-digested genomic DNA template (input) was used as a positive control.

2.6 Statistical analysis. Qualitative analysis of CpG co-methylation was performed on the pools of rDNA clones. For pooling, clones with unique $\mathrm{mCpG}$ patterns were selected from each individual. In order to minimize biases due to differences in individual's unique read numbers, 
the equal number of reads was randomly selected for each subject and pooled together within each group. Then, for the each pair of $\mathrm{CpG}\left(\mathrm{CpG}_{\mathrm{x}}\right.$ and $\left.\mathrm{CpG}_{\mathrm{y}}\right)$, the empirical frequencies of the methylation were calculated using a Python script. The possible distinct patterns for a pair could be $11,10,01$, and 00 when 1 or 0 denotes a methylation event or no methylation event, respectively. If the empirical frequencies of the patterns for a given pair $(\mathrm{X}, \mathrm{Y})$ are $p_{11}, p_{10}, p_{01}$ and $p_{00}$ then the pair $(\mathrm{X}, \mathrm{Y})$ is said to (approximately) admit the probability distribution $P(\mathrm{X}=1$, $\mathrm{Y}=1)=p_{11}, P(\mathrm{X}=1, \mathrm{Y}=0)=p_{10}, P(\mathrm{X}=0, \mathrm{Y}=1)=p_{01}$ and $P(\mathrm{X}=0, \mathrm{Y}=0)=p_{00}$. Based on the empirical frequency values we calculated the methylation correlation coefficient ( $m c c$ ) for each group, based on the following formula:

$$
m c c=\frac{p_{11}-\left(p_{01}+p_{11}\right)\left(p_{10}+p_{11}\right)}{\sqrt{\left(p_{11}+p_{01}\right)\left(1-p_{11}-p_{01}\right)\left(p_{11}+p_{10}\right)\left(1-p_{11}-p_{10}\right)}}
$$

In order to obtain the distributional properties of $m c c$ in terms of the read pools, the mcc values were re-sampled ten thousand times with different pools of randomly selected reads. For the analysis of the hierarchical clustering patters, in order to de-noisify the data, the thresholded dissimilarity statistic $\mathrm{d}(\mathrm{X}, \mathrm{Y})=1-\max ($ Thres, $m c c)$ was used to define the tree distances. The threshold value Thres=0.5 was used, as suggested by the 10 -fold cross-validation procedure. Overall, the similarity pattern analysis based on $m c c$ followed the previously reported approach (Rempala et al. 2011). The permutation test was used to determine similarity of the clusters between donor groups. The non-parametric Mann-Whitney test ( $u$-test) or Kruskall-Wallis ANOVA were used for comparisons of average $\mathrm{CpG}$ methylation between the groups, $\mathrm{mCpG \# 23}$ analysis using HpaII-qPCR or NGBS and $\mathrm{CpG}$ methylation at various positions across the promoter. 


\section{Results and Discussion}

3.1 Analysis of rDNA promoter methylation by NGBS. A NGBS approach was applied to study the effects of neurodegeneration on rDNA promoter methylation in a large number of individual rDNA units (Fig. 1). The 454 technology was chosen as at present this is the only NGS platform capable of sequencing all $26 \mathrm{CpG}$ sites of the rDNA promoter region in a single read (196 bp Fig. 1A, B). To validate NGBS analysis, a methylation-sensitive restriction endonuclease HpaII and qPCR were used to determine methylation of a specific rDNA promoter CpG site that was part of a HpaII cleavage site (CpG\#23, position -10 relative to the transcription start site, Fig. 1A, C). Donors included patients with MCI, AD and AT as well as age matched controls (Table 1).

Overall 106,157 reads were obtained including 62,096 full-length sequences. As the aim of the study was to analyze methylation of the entire promoter at the individual rDNA unit level, further analysis was focused on full-length reads only. In such clones, overall $\mathrm{CpG}$ methylation rate was $35.72 \%$. In addition, on average $7.77 \%$ of reads were non-methylated. Importantly, conversion errors were unlikely affecting these results as average $\mathrm{CpH}$ non-conversion among all full rDNA promoter reads was $0.42 \%$. Such a non-conversion rate suggests at least $99.58 \%$ conversion efficiency which matches the optimal conversion efficiency of at least $99.5 \%$ as specified by the manufacturer of the bisulfite conversion kit that was used in this study (EZ DNA Methylation-Direct Kit, Zymo Research).

Despite equal representation of individual samples in the pooled PCR product, great variability in number of NGS reads was observed between samples (Fig. 2A). While average 
number of full-length reads per individual was $1,348 \pm 1,509$, the individual values ranged from 64 to 8,261 . Such a variability suggested non-quantitative representation of the rDNA unit pool. Therefore, an alternative method, the HpaII-qPCR assay was used to quantitatively determine rDNA promoter methylation at $\mathrm{CpG} \# 23$ (Fig. 2B). CpG\#23 was significantly hypermethylated in MCI (Fig. 2B). AD samples showed a hypermethylation trend that was not significant (Fig. 2B). Such findings are in concert with our prior observations that in the human rDNA promoter, hypermethylation of all CpGs including CpG\#23 is stronger in MCI than AD (Pietrzak et al. 2011). No changes of methylation at CpG\#23 were observed in AT (Fig. 2B). Importantly, when NGBS- and HpaII-obtained values of methylation at $\mathrm{CpG} \# 23$ were compared for each sample, only a weak correlation was observed (Fig. 2C). Conversely, strength of correlation improved when analysis was done only on those samples with a greater read representation $\left(R^{2}=0.59\right.$ for 13 samples with number of reads $>$ than the all sample average of 1,348). Therefore, NGBS may be quantitative only for those samples that are represented by a high number of reads. Hence, at least for the 454 platform that produces less reads than the Illumina technology, multiple NGS runs or limited multiplexing may be required to achieve sufficient number of reads for reliable quantifications.

Given these limitations it was not surprising that NGBS data from all samples failed to reveal any significant differences of $\mathrm{CpG} \# 23$ methylation in $\mathrm{MCI}, \mathrm{AD}$ or $\mathrm{AT}$ (data not shown). Likewise, no effects on overall density of methylation across the entire rDNA promoter were observed (data not shown). Unfortunately, the number of high read samples was too low for meaningful comparisons between groups (Fig. 2A). 
3.2 CpG methylation patterns of the human rDNA promoter. Although the applied NGBS approach did not result in quantitative data, it provided an unprecedentedly broad insight into rDNA promoter methylation at the level of individual rDNA units. Such data offered a unique opportunity to perform a qualitative analysis of rDNA promoter methylation patterns.

To develop a qualitative analysis of $\mathrm{CpG}$ methylation patterns in the rDNA promoter, reads with unique sequences at $\mathrm{CpG}$ sites were selected as representatives of distinct $\mathrm{CpG}$ methylation patterns. As with the number of all full length reads, the number of unique $\mathrm{CpG}$ reads was highly variable between samples (Fig. 3A). Therefore, to avoid possible biases due to uneven representation, random drawing was used to construct pools of rDNA units for each donor group with even representation of each sample (Fig. 3B). The size of a sample with the lowest number of unique reads (old control donor \#8, 23 unique reads) determined drawing size from all other samples. The CpG methylation patterns were analyzed in such randomly constructed pools for each group of donors. To obtain representative trends, the pooling-analysis procedure was repeated 10,000 times.

For all groups there was a remarkable consistency of methylation frequencies at specific CpG sites (Fig 3C-D). When each group was treated as an independent sample, a highly significant effect of $\mathrm{CpG}$ site location on its methylation frequency was detected (KruskallWallis ANOVA, $\mathrm{p}<0.0001, \mathrm{n}=5$ ). More than 50\% methylation was observed at $\mathrm{CpG}$ sites \#9, 20 and 21 (compare to $37.6 \pm 12 \%$ average $\mathrm{CpG}$ methylation for all groups). For $\mathrm{CpG} \# 9$ and 20 the hypermethylation was significant when compared to a nearest $\mathrm{CpG}$ site with above average methylation (CpG\#10 and CpG\#19, respectively, $\mathrm{p}<0.05, u$-test). Of note, CpG\#23 whose methylation can be easily studied using the HpaII-qPCR assay was the fourth most methylated 
site of the rDNA promoter with $49.8 \pm 4.1 \%$ methylation. In contrast, less than $25 \%$ methylation was found at CpGs\#2, 3, 5 and 25. Their hypomethylation was significant when compared to a

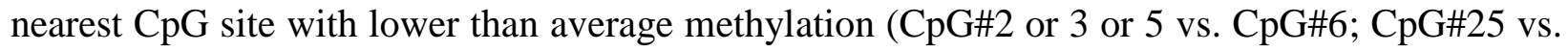
CpG\#24, p<0.05, $u$-test). Such differences in frequency of methylation may indicate relatively greater importance of the hypermethylated CpGs in rDNA silencing.

CpG co-methylation patterns were evaluated by calculating the methylation correlation coefficient ( $m c c$ ) for each pair of $\mathrm{CpG}$ sites from the rDNA promoter. Mcc reflects the probability of co-methylation at two given sites with a correction for the expected random comethylation as determined by the empirical frequencies of methylation at each individual site (see Material and Methods for details). For instance, mcc equals 0 when two sites are comethylated at a frequency that is fully explained by the empirical frequencies of their individual methylations (e.g. co-methylation occurs in a random manner). Conversely, mcc equals 1 when two sites are methylated together but never alone (e.g. methylation occurs at both sites together but never at either site alone). Mcc equals -1 when either site is always methylated but they are never co-methylated (e.g. methylation at either site precludes their co-methylation). Thus, positive values of $m c c$ would indicate a bias towards co-methylation; negative values of $m c c$ would suggest that mono-methylations are preferred while co-methylation is avoided.

In all 5 groups, $m c c$ values for all pairs of $26 \mathrm{CpG}$ sites were positive indicating a bias towards co-methylation across the entire rDNA promoter (Fig. 4A-B). Average mcc value was 0.31, 0.28, 0.26, 0.27, or 0.23 for young control, AT, old control, MCI, or AD, respectively (Fig. 4A). The apparent age- or neurodegeneration-dependent decreases of the average mcc were 
significant (Fig. 4A). These findings suggest that aging and/or neurodegeneration may be associated with increasingly random $\mathrm{CpG}$ methylation of the rDNA promoter.

However, similarities of $m c c$ distribution were also obvious in all analyzed groups. Thus, in all of them, maximal $m c c$ values ranging from $0.54-0.63$ were observed for pairs that were formed by CpG\#20, 21 and/or 23 (Fig. 4B). These CpGs are located in the core promoter (CP, positions -49 to 20) 5 ' to the transcription initiation site (positions $-31,-26$ and -10 , respectively). Moreover, within each group of donors, hierarchical clustering analysis of $m c c$ patterns revealed that pairs of CpGs\#20, 21 and/or 23 were significantly more co-methylated than any other CpGs (Fig. 4C). Therefore, relatively higher frequency of methylation at CpG\#20, 21, and/or 23, a bias towards their co-methylation and their location in the critical region of the rDNA promoter suggest that they may play an important role in rDNA silencing. That may simply be due to the fact that $\mathrm{CP}$ is the most critical regulatory region for rDNA transcriptional activity (Haltiner $e t$ al. 1986). In addition, strong co-methylation may also indicate that in the $\mathrm{CP}$, at least two $\mathrm{CpG}$ sites have to be methylated to effectively suppress transcription. Indeed, selective methylation at CpG\#23 had a greater, yet partial transcription-inhibitory effect than that at the non-CP CpG\#8 (Ghoshal et al. 2004). In addition, while RNA-Polymerase-1 binding was occasionally detected in rDNA units containing mCpGs in the upstream control element UCE (as defined at positions 186 to -51), CP methylation was never observed in these active genes (Brown \& Szyf 2007). Hence, $\mathrm{CP}$ appears to be critical for mCpG-mediated silencing of human rDNA. However, future studies are needed to confirm this possibility in the context of human tissues. For instance, correlation analysis between CP/UCE methylation status and rDNA promoter occupancy by active Pol1 and/or its association with active/inactive histone marks could establish the most 
direct link between methylation patterns and rDNA transcription. Post mortem samples such as those used in our current work may not be optimal for this purpose as nucleolar transcription is expected to be highly sensitive to post-mortem ischemia (Murayama et al. 2008). Conversely, temporal lobe fragments that are removed during anti-epileptic surgeries could provide better insight into rDNA transcription in the human brain.

Hierarchical clustering analysis also revealed some differences in patterns of $\mathrm{CpG}$ comethylation between the pooled rDNA clones from the investigated groups (Fig. 4C, Table 2). Thus, CpG\#21 and 23 showed strong clustering in elderly control- and AD- but not in MCI group. In the latter group, CpG\#20 and 21 and to a lesser extent 23 were closely clustered. Such a pattern resembled younger controls and AT patients. Consistently, pattern clustering of elderly controls was significantly different than that of younger control- AT-, or MCI group (Table 2). Importantly, age of AT- or MCI donors matched that of young- or elderly controls, respectively (Table 1). Therefore, these results suggest that aging may be a factor that affects co-methylation of the rDNA promoter CpGs and that MCI may be associated with a "rejuvenation" of the comethylation pattern(s). Finally, stronger trends towards clustering in pools of younger donors provide yet another indicator of more random methylation in elderly. These differences suggest that in the human brain rDNA promoter, $\mathrm{CpG}$ methylation becomes increasingly disorganized as the brain ages and/or is confronted with a neurodegenerative disease. However, any differences between pooled samples have to be interpreted with caution as they may misrepresent individual trends. 


\subsection{Non-CpG methylation in the human rDNA promoter. Non-converted Cs were}

occasionally observed at $\mathrm{CpH}$ locations (Fig. 5A). The observed mCpH events were unlikely to be all artifacts of non-conversion errors. For instance, a $\mathrm{CpH}$ at position -94 was found nonconverted in $0.77 \%$ of all full length reads from young controls. That is a higher rate of $\mathrm{CpH}$ non-conversion than $0.42 \%$ for an average $\mathrm{CpH}$ site in the rDNA promoter. Moreover, for this site above average non-conversion was also found in at least three other groups (old control-, MCI-, and AD). Similar criteria were met by 8 other $\mathrm{CpH}$ sites (Fig. 5A). Hence, CpHs at these locations are likely targets of methylation.

To confirm presence of $\mathrm{mCpH}$ in the rDNA promoter, the methylation-sensitive restriction endunclease PspGI was used. Methylation at the $\mathrm{CpH}-94$ would prevent a specific PspGImediated cleavage of a genomic DNA template; such a PspGI resistance could then be detected by qPCR (Fig. 5B). Indeed, partial PspGI resistance was detected in a pooled DNA sample from all control donors (Fig. 5C). Hence, mCpH occurs in the rDNA promoter at position -94 and may also occur at several other sites in this region (Fig. 5D).

$\mathrm{CpH}$ methylation has been commonly found in pluripotent cells and in the brain (Lister et al. 2009, Lister et al. 2013, Zeng et al. 2012). While functional significance of CpH methylation is not clear, it is enriched in repressed genes suggesting its possible inhibitory role in regulating gene expression (Lister et al. 2013). Importantly, changes in $\mathrm{CpH}$ methylation have been previously documented in AD-relevant genes (Fuso et al. 2011). In addition, it has been proposed that $\mathrm{mCpC}$ sites (such as the $\mathrm{mCpH}$ at position -94 of the human rDNA) recruit demethylation machinery to both $\mathrm{CpH}$ and $\mathrm{CpG}$ sites of differentiation-regulated genes (Fuso et 
al. 2010). Hence, $\mathrm{CpHs}$ may work together with $\mathrm{CpGs}$ to regulate rDNA silencing and/or fine tune transcription of active rDNA genes. However, as in our sample sets methylation of $\mathrm{CpH}$ sites from the rDNA promoter was relatively uncommon it is unlikely that $\mathrm{mCpH}$ plays a major role in epigenetic regulation of rDNA in the adult and/or aging human brain.

3.4 Conclusions. NGBS offered a unique insight into methylation patterns of individual rDNA promoters in the human brain. In that region, (i) $\mathrm{CpGs}$ at various positions were not equally methylated, (ii) CpG co-methylation was widely spread, (iii) a hypermethylation/comethylation hot-spot was present in the core promoter, and, finally, (iv) $\mathrm{CpH}$ methylation was relatively rare. While these conclusions are consistent with a view that the average density of methylated CpGs in the rDNA promoter may determine human rDNA transcription (Brown \& Szyf 2007, Ghoshal et al. 2004), they also suggest that methylation at certain CpG sites may have a relatively greater regulatory influence over human rDNA than others.

\section{Acknowledgments}

We are grateful for the research volunteers that contributed to this work. This work was supported by NIH (NS073584-01 and 8P30GM103507 to MH; CA152158 to GR; AG028383 to PN), NSF (IOS1021860 to MH; DMS1318886 to GR), The Commonwealth of Kentucky Challenge for Excellence, and the Kentucky Spinal Cord and Head Injury Research Trust. Some samples (young controls and AT) were provided by the NICHD Brain and Tissue Bank for Developmental Disorders and NICH Contract \#HHSN275200900011C, Ref. No. NO1-HD-90011. The authors wish to thank Dr. Scott A. Myers for critical reading of the manuscript and Ms. Jing-Juan Zheng for excellent technical assistance. 


\section{References}

Brown, S. E. and Szyf, M. (2007) Epigenetic programming of the rRNA promoter by MBD3. Molecular and cellular biology, 27, 4938-4952.

Ding, Q., Markesbery, W. R., Cecarini, V. and Keller, J. N. (2006) Decreased RNA, and Increased RNA Oxidation, in Ribosomes from Early Alzheimer's Disease. Neurochem Res.

Ding, Q., Markesbery, W. R., Chen, Q., Li, F. and Keller, J. N. (2005) Ribosome dysfunction is an early event in Alzheimer's disease. J Neurosci, 25, 9171-9175.

Freed, E. F., Bleichert, F., Dutca, L. M. and Baserga, S. J. (2010) When ribosomes go bad: diseases of ribosome biogenesis. Mol Biosyst, 6, 481-493.

Fuso, A., Ferraguti, G., Grandoni, F., Ruggeri, R., Scarpa, S., Strom, R. and Lucarelli, M. (2010) Early demethylation of non-CpG, $\mathrm{CpC}$-rich, elements in the myogenin 5'-flanking region: a priming effect on the spreading of active demethylation. Cell Cycle, 9, 3965-3976.

Fuso, A., Ferraguti, G., Scarpa, S., Ferrer, I. and Lucarelli, M. (2015) Disclosing bias in bisulfite assay: MethPrimers underestimate high DNA methylation. PLoS One, 10, e0118318.

Fuso, A., Nicolia, V., Pasqualato, A., Fiorenza, M. T., Cavallaro, R. A. and Scarpa, S. (2011) Changes in Presenilin 1 gene methylation pattern in diet-induced B vitamin deficiency. Neurobiol Aging, 32, 187-199.

Ghoshal, K., Majumder, S., Datta, J., Motiwala, T., Bai, S., Sharma, S. M., Frankel, W. and Jacob, S. T. (2004) Role of human ribosomal RNA (rRNA) promoter methylation and of methyl-CpG-binding protein MBD2 in the suppression of rRNA gene expression. $J$ Biol Chem., 279, 6783-6793. Epub 2003 Nov 6710. 
Guetg, C., Lienemann, P., Sirri, V., Grummt, I., Hernandez-Verdun, D., Hottiger, M. O., Fussenegger, M. and Santoro, R. (2010) The NoRC complex mediates the heterochromatin formation and stability of silent rRNA genes and centromeric repeats. The EMBO journal, 29, 2135-2146.

Haltiner, M. M., Smale, S. T. and Tjian, R. (1986) Two distinct promoter elements in the human rRNA gene identified by linker scanning mutagenesis. Molecular and cellular biology, $\mathbf{6}$, 227-235.

Hetman, M. and Pietrzak, M. (2012) Emerging roles of the neuronal nucleolus. Trends Neurosci, 35, 305-314.

Lister, R., Mukamel, E. A., Nery, J. R. et al. (2013) Global epigenomic reconfiguration during mammalian brain development. Science, 341, 1237905.

Lister, R., Pelizzola, M., Dowen, R. H. et al. (2009) Human DNA methylomes at base resolution show widespread epigenomic differences. Nature, 462, 315-322.

Lutsik, P., Feuerbach, L., Arand, J., Lengauer, T., Walter, J. and Bock, C. (2011) BiQ Analyzer HT: locus-specific analysis of DNA methylation by high-throughput bisulfite sequencing. Nucleic Acids Res, 39, W551-556.

McStay, B. and Grummt, I. (2008) The epigenetics of rRNA genes: from molecular to chromosome biology. Annu Rev Cell Dev Biol., 24, 131-157.

Murayama, A., Ohmori, K., Fujimura, A. et al. (2008) Epigenetic control of rDNA loci in response to intracellular energy status. Cell., 133, 627-639.

Nelson, P. T., Jicha, G. A., Schmitt, F. A., Liu, H., Davis, D. G., Mendiondo, M. S., Abner, E. L. and Markesbery, W. R. (2007) Clinicopathologic correlations in a large Alzheimer 
disease center autopsy cohort: neuritic plaques and neurofibrillary tangles "do count" when staging disease severity. J Neuropathol Exp Neurol, 66, 1136-1146.

Pietrzak, M., Rempala, G., Nelson, P. T., Zheng, J. J. and Hetman, M. (2011) Epigenetic silencing of nucleolar rRNA genes in Alzheimer's disease. PLoS One, 6, e22585.

Rempala, G. A., Seweryn, M. and Ignatowicz, L. (2011) Model for comparative analysis of antigen receptor repertoires. Journal of theoretical biology, 269, 1-15.

Slomnicki, L. P., Pietrzak, M., Vashishta, A. et al. (2016) Requirement of neuronal ribosome synthesis for growth and maintenance of the dendritic tree. J Biol Chem.

Zeng, J., Konopka, G., Hunt, B. G., Preuss, T. M., Geschwind, D. and Yi, S. V. (2012) Divergent whole-genome methylation maps of human and chimpanzee brains reveal epigenetic basis of human regulatory evolution. Am J Hum Genet, 91, 455-465. 


\section{Figure Legends}

Figure 1. NGBS and HpaII-qPCR strategies to analyze rDNA methylation of the human rDNA promoter. $\boldsymbol{A}$, Sequence of the human rDNA promoter region containing all $26 \mathrm{CpG}$ methylation sites (marked in grey). The HpaII site is underlined, numbers preceded by + or indicate position relative to transcription start site (arrow). $\boldsymbol{B}$, A flow chart depicting the NGBS analysis. The bisulfite-converted rDNA promoter fragment was amplified using primers containing barcodes (MID). The barcoded DNA from all patients was pooled and sequenced using 454 technology. $C$, Location of the amplicons used for HpaII-qPCR analysis of mC content at the CpG site \#23 of the human rDNA promoter.

Figure 2. Analysis of rDNA promoter methylation in post-mortem samples of the parietal cortex. Donors included individuals with MCI, AD, AT and age-matched controls. $\boldsymbol{A}$, Number of reads covering the entire rDNA promoter fragment that were obtained for each subject. Note great variability in representation of individual subjects among the reads. B, Quantitative analysis of the rDNA methylation at CpG\#23 (position -10 from the transcription start site) by

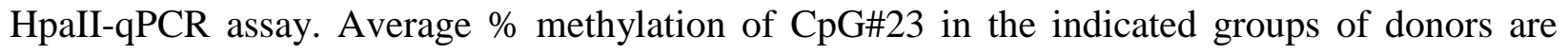
depicted on the graphs; individual values are represented by dots; mean values are indicated by the lines intersecting the error bars (SEM). Significant hypermethylation was present in MCI patients as compared to age-matched controls ( $u$-test $p$ value is indicated); no significant differences were detected for other comparisons. $\boldsymbol{C}$, Regression analysis to compare NGBS data

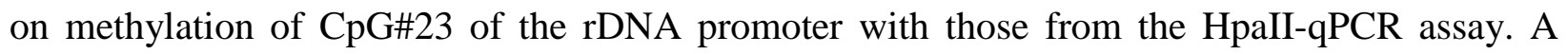
weak correlation between the two data sets suggests that uneven representation of subjects in 
NGBS reads makes them an unreliable source of quantitative information on individual $\mathrm{mCpG}$ content.

\section{Figure 3. Qualitative analysis of $\mathrm{CpG}$ methylation patterns of the rDNA promoter. $A$,} Number of reads with unique sequences per each analyzed individual. $\boldsymbol{B}$, A flow chart depicting analysis of $\mathrm{CpG}$ methylation patterns among reads with unique sequences. To improve equality of representation, random drawing of the same number of reads from each individual was performed for each group of subjects creating groups-specific pools of rDNA clones. Frequency of methylation $(f)$ for each $\mathrm{CpG}$ site and a methylation correlation co-efficient for each pair of CpG sites $(m c c)$ were calculated to compare methylation patterns. Such analyses were repeated on 10,000 randomly selected pools (see Materials and Methods and Results for more details). $\boldsymbol{C}$ $D$, Methylation frequency analysis of $\mathrm{CpG}$ sites throughout the rDNA promoter in pools of unique reads from each group of subjects. Frequencies of mCpGs for each donor pool are depicted in $\mathrm{C}$; average $\mathrm{mCpG}$ frequencies $\pm \mathrm{SD}$ of all five donor pools are depicted in $\mathrm{D}$. Note that across the rDNA promoter, distribution of $\mathrm{mCpG} f$ values is similar for all groups of donors. When each group was treated as an independent sample, $\mathrm{CpG}$ position had a highly significant effect on $\mathrm{mCpG}$ frequency (Kruskall-Wallis ANOVA, $\mathrm{p}<0.0001$ ).

Figure 4. CpG co-methylation patterns of the rDNA promoter. $\boldsymbol{A}$, The general $\mathrm{CpG}$ comethylation trend in the rDNA promoter appears to be affected by age and progressing neurodegeneration. Average $m c c$ values of all $\mathrm{CpG}$ pairs are depicted; error bars are SEM; NS, $\mathrm{p}>0.05 ; *, \mathrm{p}<0.05, * * *, \mathrm{p}<0.001$ ( $u$-test). The declining $m c c$ values in old control- or in AT or AD groups may suggest an increasingly disorganized methylation pattern as the brain ages 
and/or is confronted with neurodegeneration. $\boldsymbol{B}$, Heat maps of methylation correlation coefficients $(m c c)$ for each $\mathrm{CpG}$ pair of the rDNA promoter. Many patterns appear to be similar between all donor groups. Moreover, all $m c c$ values are positive indicating that a trend towards co-methylation is present throughout the $\mathrm{CpG}$ island of the rDNA promoter. The highest values are observed for $\mathrm{CpG}$ pairs of the core promoter (CpG\#20, 21 and 23); the lowest values are observed for CpGs of the transcribed region (CpG\#24, 25 and 26). $\boldsymbol{C}$, Hierarchical clustering analysis of $\mathrm{CpG}$ co-methylation patterns as revealed by mcc. Similarity of patterns is indicated by a reduction of a relative distance between them on tree diagrams (see Materials and Methods for more details). In all five pools of rDNA clones, highly similar patterns were detected for CpGs of the core promoter including CpG\#20, 21 and/or 23. In addition, in pools from younger subjects (young controls and AT patients), additional similarities emerged including those for CpG\#17 and 18 of the upstream control element region (see text for more details; permutationbased $p$-values for comparisons of pattern clustering for each pair of donor groups are in Table 2).

Figure 5. CpH methylation of the rDNA promoter. $\boldsymbol{A}$, Reads with unconverted C identify 35 putative $\mathrm{CpH}$ methylation sites. Frequency of unconverted $\mathrm{CpH}$ reads (y axis) among all pooled full length reads for each group of donors. Yellow bars indicate $9 \mathrm{CpH}$ sites with frequency of non-conversion that was above average for all $35 \mathrm{CpH}$ sites in at least 4 of 5 analyzed groups of donors. These $9 \mathrm{CpHs}$ represent the most likely sites of non-CpG methylation. $\boldsymbol{B}-\boldsymbol{C}, \mathrm{CpH}$

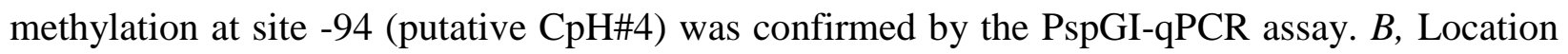
of an amplicon that was used for the qPCR determination of genomic DNA cleavage by the restriction endonuclease PspGI; positions relative to transcription start site are indicated by the 
numbers; dotted lines correspond to PCR primers; sequence of the PspGI site that is sensitive to methylation at position -94 (asterisk) is in black uppercase; arrowheads indicate the cuts. $\boldsymbol{C}$, qPCR of pooled genomic DNA from control groups that was treated with PspGI revealed that there was a fraction of a template that was resistant to cleavage by PspGI but not the methylation-insensitive $\mathrm{MspI}$. $\boldsymbol{D}, \mathrm{CpG}$ and $\mathrm{CpH}$ methylation in the human rDNA promoter. In a human rDNA unit, the location of the promoter region is indicated by a white box; rRNA coding regions correspond to grey boxes. Location of each methylated $\mathrm{CpG}$ - or likely methylated $\mathrm{CpH}$ site is indicated on the promoter sequence; grey numbers above the sequence indicate $\mathrm{CpG}$ sites; black numbers under the sequence indicate $\mathrm{CpH}$ sites that are also framed in red. The confirmed $\mathrm{CpH}$ site \#4 (position -94) is pointed by an asterisk. Numbers preceded by + or - indicate position relative to transcription start site (arrow). 
Table 1. Demographic and neurocognitive profile of AD and AT patients and age-matched controls.

\begin{tabular}{|c|c|c|c|c|c|}
\hline & $\begin{array}{c}\text { Control } \\
\text { (old) }\end{array}$ & MCI & AD & $\begin{array}{c}\text { Control } \\
\text { (young) }\end{array}$ & AT \\
\hline $\begin{array}{c}\text { Number of } \\
\text { subjects }\end{array}$ & 10 & 10 & 10 & 9 & 7 \\
\hline Age (years) & $82.6 \pm 1.77^{\mathrm{c}}$ & $86.6 \pm 1.86^{\mathrm{c}}$ & $80.1 \pm 1.68^{\mathrm{c}}$ & $26.44 \pm 3.51^{\mathrm{c}}$ & $27.85 \pm 3.95^{\mathrm{c}}$ \\
\hline Sex (F:M) & $4: 6$ & $7: 3$ & $3: 7$ & $5: 4$ & $3: 4$ \\
\hline Brain weight (g) $^{\text {Bun }}$ & $1234 \pm 36.54^{\mathrm{c}}$ & $1150 \pm 30.48^{\mathrm{c}}$ & $1167 \pm 45.0^{\mathrm{c}}$ & $\mathrm{NA}$ & $\mathrm{NA}$ \\
\hline PMI (h) $^{\mathrm{a}}$ & $2.23 \pm 0.20^{\mathrm{c}}$ & $3.05 \pm 0.36^{\mathrm{c}}$ & $3.2 \pm 0.15^{\mathrm{c}}$ & $7 \pm 0.47^{\mathrm{c}}$ & $8 \pm 2.12^{\mathrm{c}}$ \\
\hline MMSE $^{\mathrm{b}}$ & $28.8 \pm 0.25^{\mathrm{c}}$ & $22.33 \pm 1.84^{\mathrm{c}}$ & $13.62 \pm 1.94^{\mathrm{c}}$ & $\mathrm{NA}$ & $\mathrm{NA}$ \\
\hline
\end{tabular}

Footnotes: ${ }^{a}$ PMI, Post Mortem Interval; ${ }^{b}$ MMSE, Minimental State Examination score; ${ }^{c}$ Mean \pm Standard Error of the Mean (SEM). 
Table 2. Comparisons of co-methylation pattern clusters for pooled rDNA clones from different groups of donors.

\begin{tabular}{|c|c|c|c|c|}
\hline MCI & $0.029^{a}$ & & & \\
\hline$A D$ & $0.568^{a}$ & $0.368^{\mathrm{a}}$ & & \\
\hline Control (young) & $0.025^{a}$ & $1.000^{\mathrm{a}}$ & $0.343^{a}$ & \\
\hline \multirow[t]{2}{*}{ AT } & $0.001^{a}$ & $0.850^{\mathrm{a}}$ & $0.029^{a}$ & $0.912^{a}$ \\
\hline & $\begin{array}{l}\text { Control } \\
\text { (old) }\end{array}$ & MCl & $A D$ & $\begin{array}{l}\text { Control } \\
\text { (young) }\end{array}$ \\
\hline
\end{tabular}

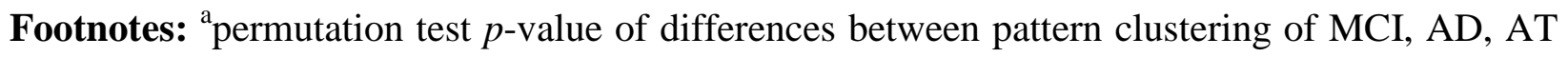
and age-matched control; $p$ values smaller than 0.05 are in italics. 


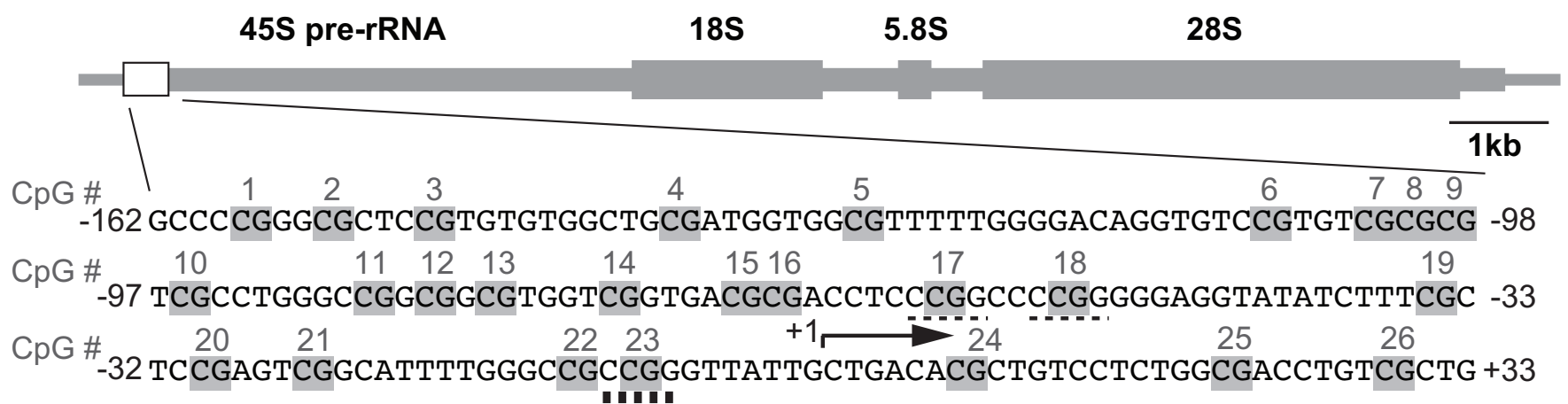

PCR amplification of BS-converted DNA (barcoding)
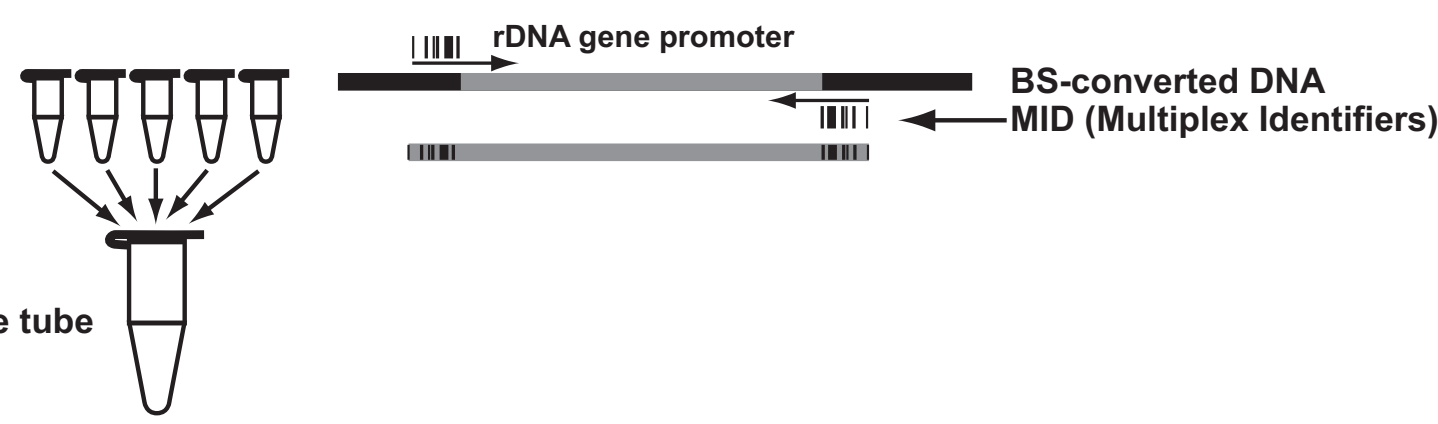

Combining PCR products in single tube

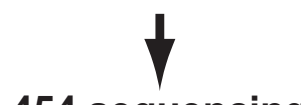

454 sequencing

$\downarrow$

Analysis

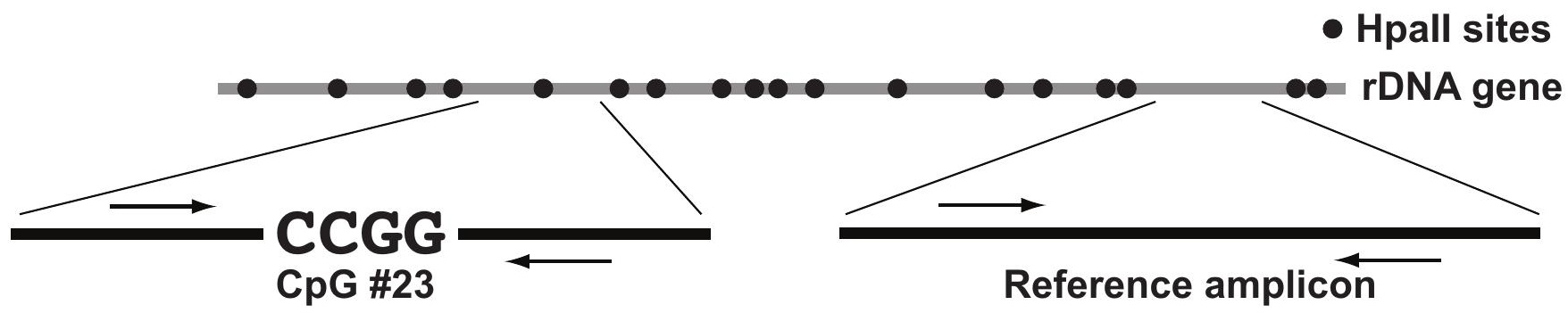


Figure 2

A
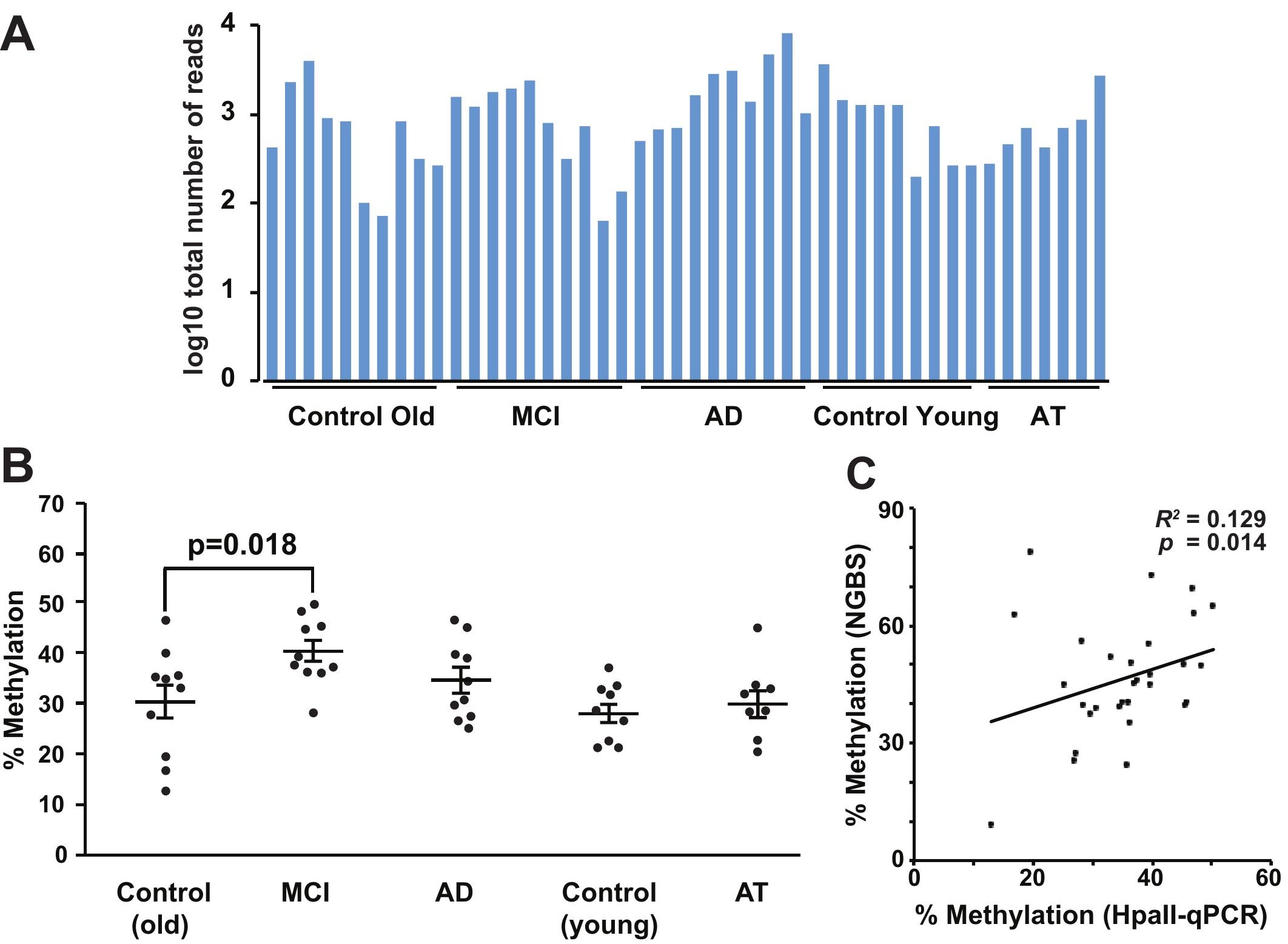
A
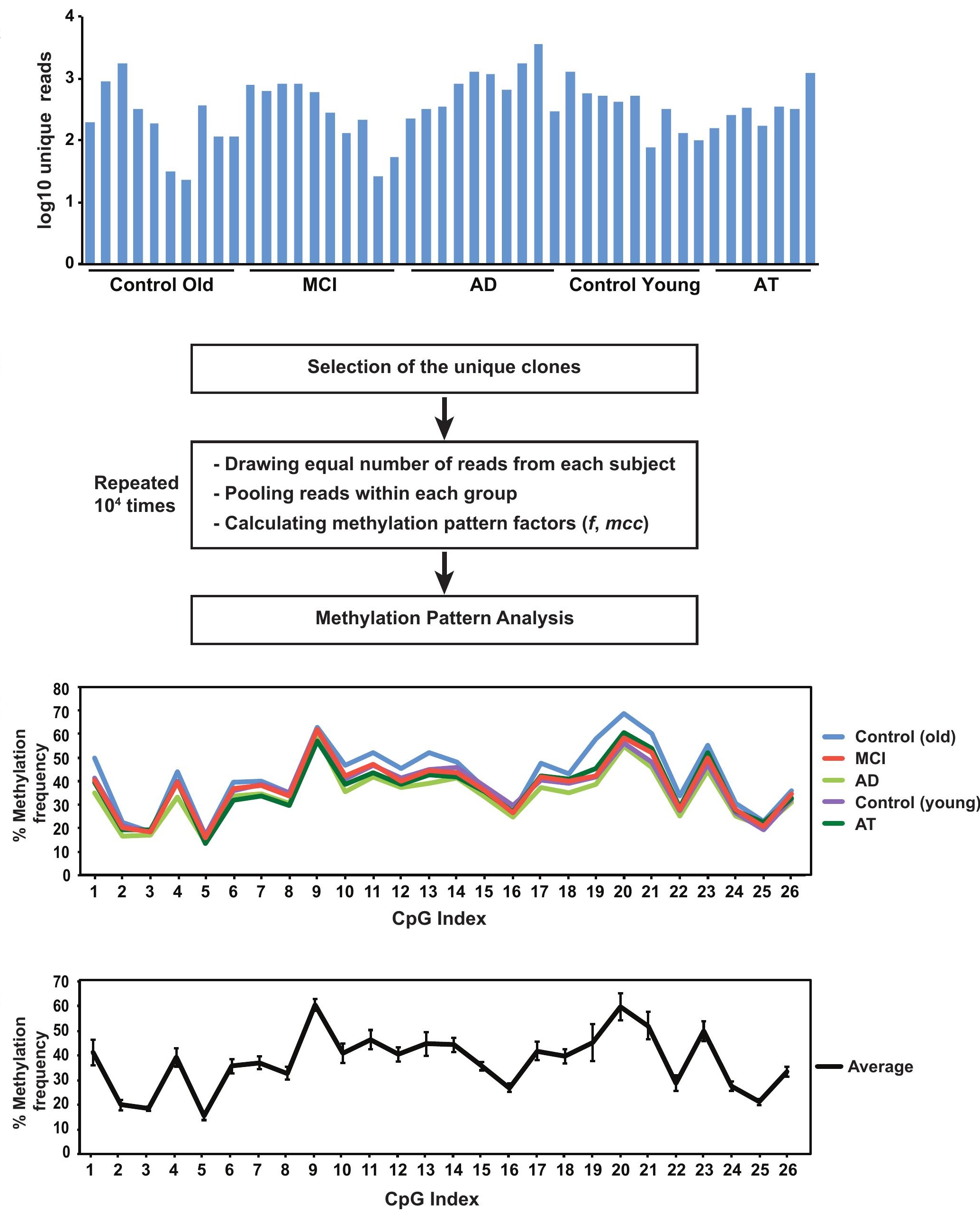

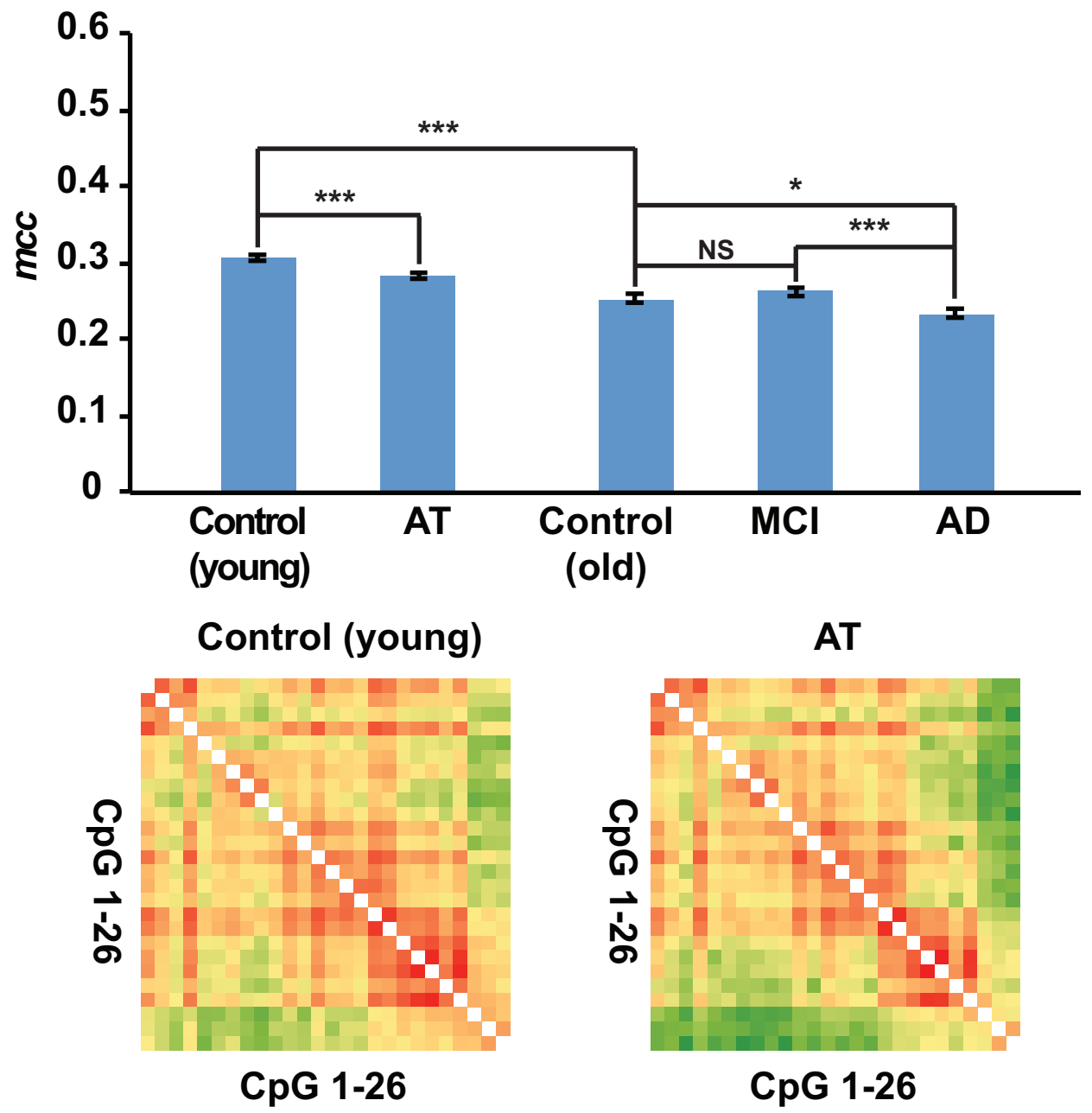

\section{CpG 1-26}

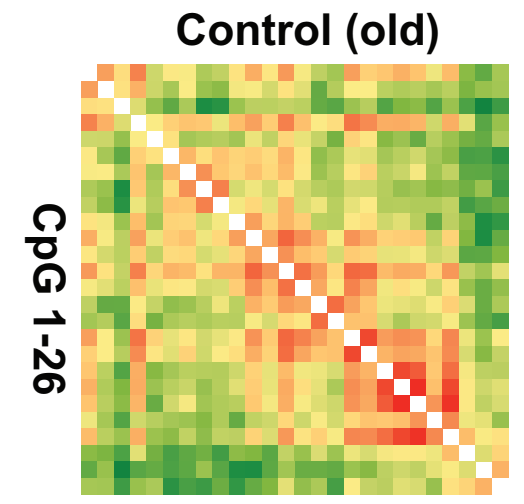

Cpg 1-26

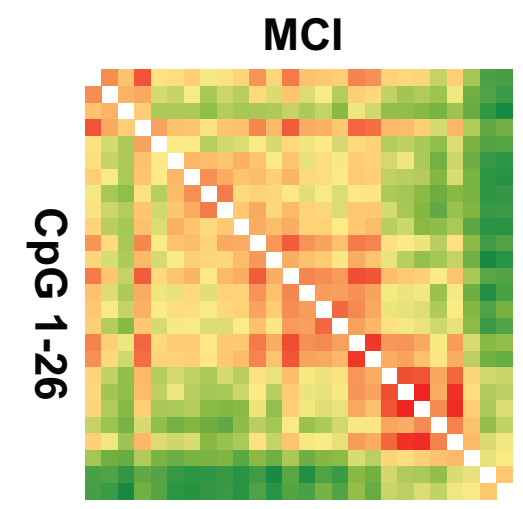

Cpg 1-26

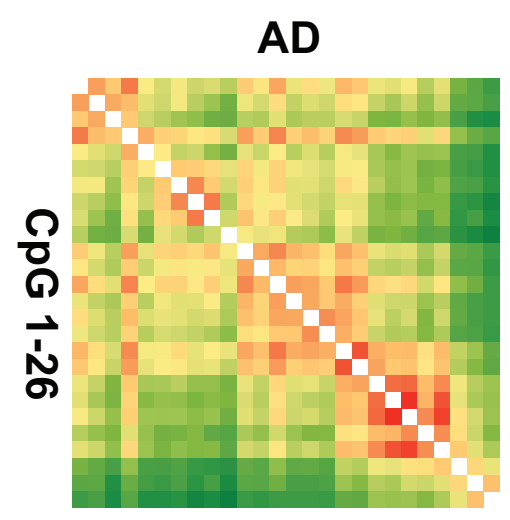

Cpg 1-26
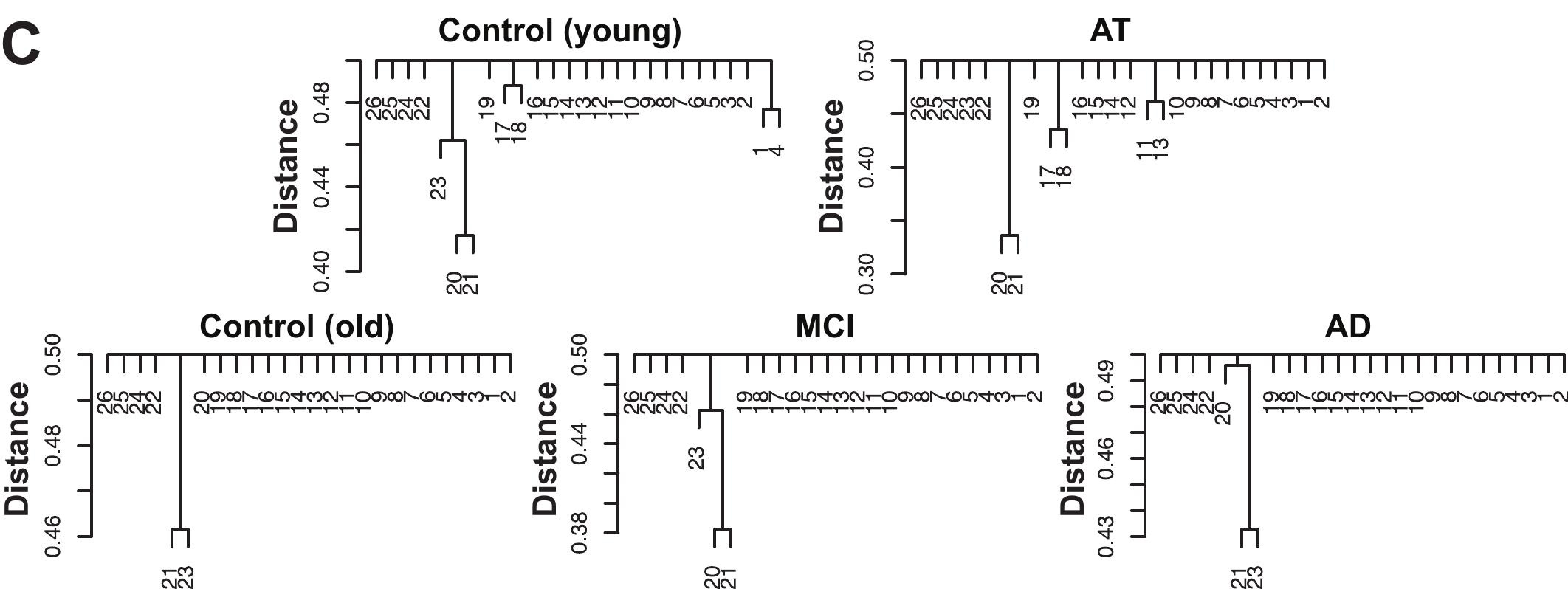


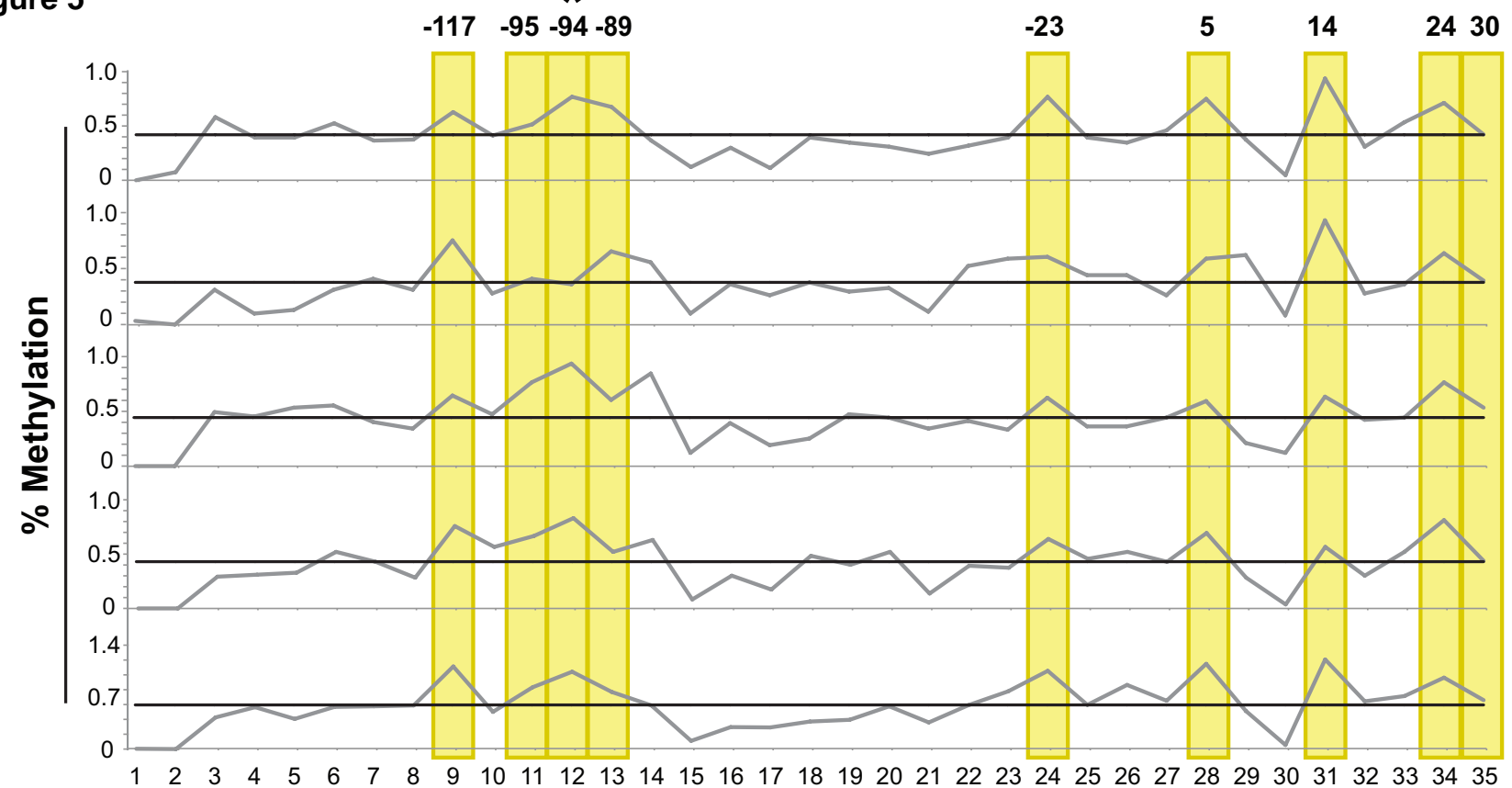

Control (young)

AT

Control (old)

$\mathrm{MCl}$

$\mathrm{CpH}$ index

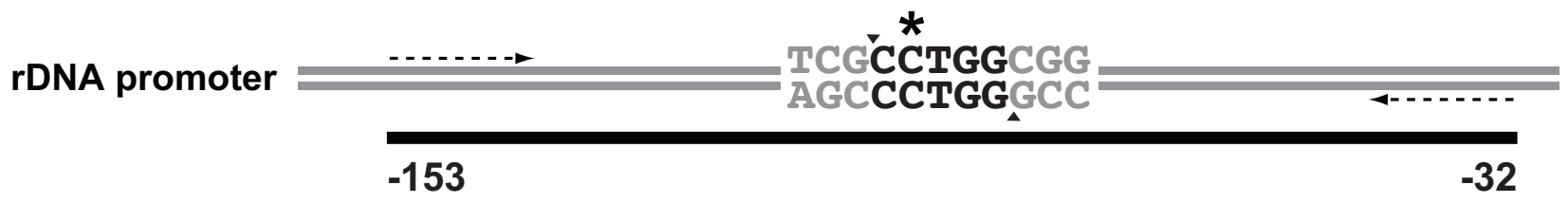

\section{Amplification Plot}

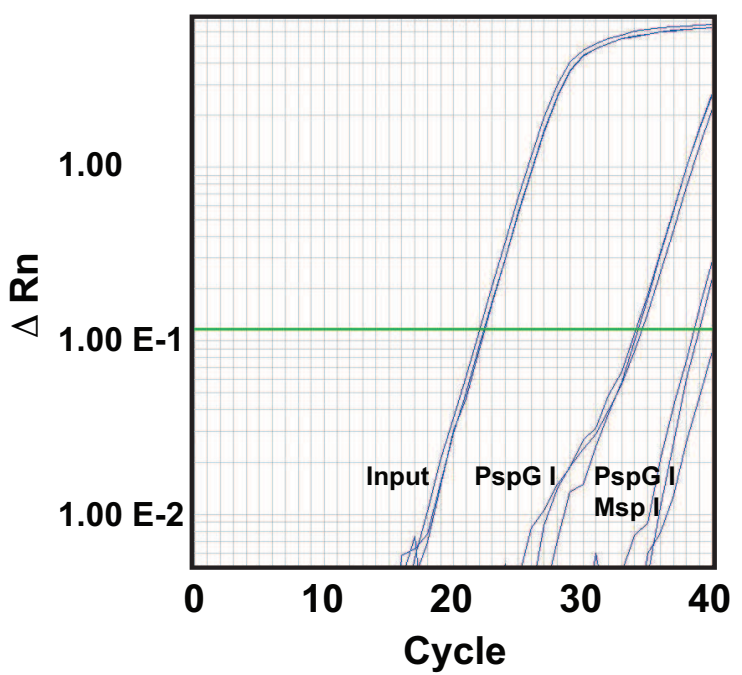

$45 \mathrm{~S}$ pre-rRNA

$18 S$

$5.8 S$

$28 S$

AD

D

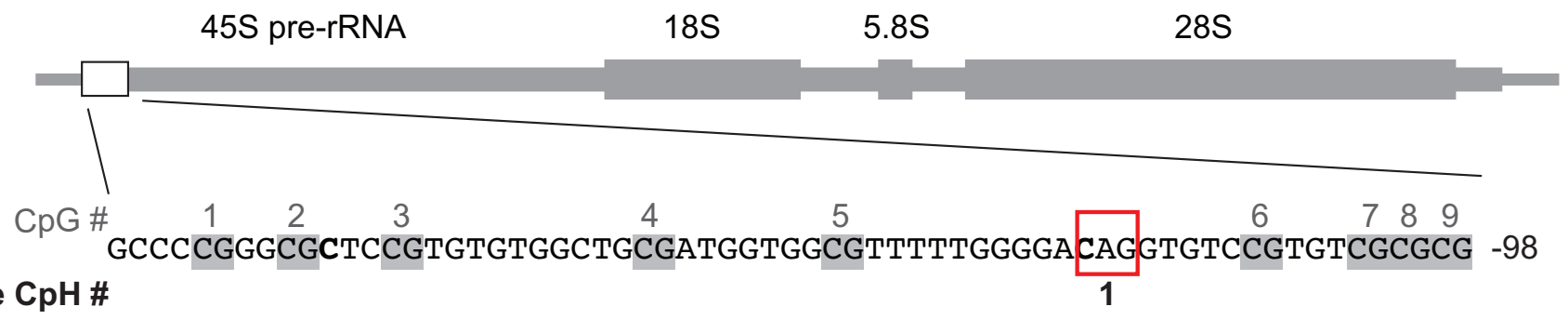
putative $\mathrm{CpH} \#$
CpG \# 10 *
$14 \quad 1516$
$17 \quad 18$

TCGCCTGGGCCGGCGGCGTGGTCGGTGACGCGACCTCCCGGCCCCGGGGGAGGTATATCTTTCGC -33 putative $\mathrm{CpH} \# \quad 2,3 \quad 4$
CpG \# 202120223
$+1 \longrightarrow 24$
25 CACTATCGTG +33 